\title{
Harmonic maps from degenerating Riemann surfaces
}

\author{
Miaomiao Zhu
}

Received: 18 March 2008 / Accepted: 23 October 2008 / Published online: 14 November 2008

(C) The Author(s) 2008. This article is published with open access at Springerlink.com

\begin{abstract}
We study harmonic maps from degenerating Riemann surfaces with uniformly bounded energy and show the so-called generalized energy identity. We find conditions that are both necessary and sufficient for the compactness in $W^{1,2}$ and $C^{0}$ modulo bubbles of sequences of such maps.
\end{abstract}

\section{Introduction}

Consider a sequence of harmonic maps from compact Riemann surfaces $\left(\Sigma_{n}, h_{n}\right)$ to a compact Riemannian manifold $(N, g)$,

$$
u_{n}: \Sigma_{n} \rightarrow N,
$$

with uniformly bounded energy $E\left(u_{n}, \Sigma_{n}\right) \leq \Lambda<\infty$.

In this paper, we study the compactness of the sequence (1.1). We shall first review some well-established analytic aspects related to this problem and then focus on the case that the domains $\Sigma_{n}$ degenerate. Our results indicate that when the topological type of the degeneration is fixed, one can associate to $\left(u_{n}, \Sigma_{n}\right)$ a sequence of quantities that characterize the asymptotic behaviour of maps in the limit process.

At first, let us consider the case that the domain surface is fixed, namely, $\Sigma_{n}=\Sigma$. The uniform energy bound $E\left(u_{n}\right) \leq \Lambda$ allows us to find a map $u: \Sigma \rightarrow N$ such that $u_{n}$ subconverges weakly to $u$. However, in general, strong convergence fails because of energy concentration at finitely many points on $\Sigma$, which are called blow-up points [29,30]. Away from these points, the convergence is strong. At these points, the "bubbling" phenomenon can occur and the concentrated energy can be captured by finitely many bubbles, i.e., non-trivial

M. Zhu was supported by IMPRS "Mathematics in the Sciences” and the Klaus Tschira Foundation.

M. Zhu $(\bowtie)$

Max Planck Institute for Mathematics in the Sciences, Inselstr. 22-26, 04103 Leipzig, Germany

e-mail: Miaomiao.Zhu@mis.mpg.de 
harmonic maps from two-spheres [29,30]. During the "bubbling" process, there are some necks joining the base $u: \Sigma \rightarrow N$ to the bubbles or one bubble to the next. Jost [13] proved that in the limit, these necks contain no energy, which means all concentrated energy is captured by the bubbles. Parker [22] showed that these necks actually converge to points in the target manifold, which means that in the limit the base and the bubbles are connected.

Next, we allow the complex structure on the domain surface to vary. In this case, consideration of the degeneration of conformal structures on a Riemann surface will be necessary. Topologically, the limit surface is obtained by collapsing finitely many simple closed curves in $\Sigma$. In the end we obtain a surface with nodes as singularities. There are two types of collapsing curves. The first is a homotopically trivial one, which corresponds to the "bubbling" near isolated singularities, for the complex structure varies in a compact region of the moduli space. The second is a homotopically nontrivial curve, which corresponds to the degeneration of the complex structure. By following the "bubbling" procedure, we can also find a limit map consisting of a union of smooth harmonic maps. However, in general, energy may get lost from some necks, and those necks will fail to converge to points, as in the explicit example given in [22].

It is worth mentioning that if, in addition, $u_{n}$ are conformal, i.e., minimal surfaces, then by the technical tools in minimal surface theory (e.g., the isoperimetric inequality and the monotonicity property, etc.), we know that in the limit, there is no energy loss and there are no necks, which gives satisfactory compactness results. For more details see, for instance, $[3,13,23]$, or [22]. An analogue is Gromov's compactness theorem for pseudo-holomorphic curves $[8,12,23,38,39]$.

By an asymptotic analysis of harmonic maps from long cylinders, Chen-Tian [4] observed that all connecting necks converge exponentially to geodesics in the target manifold. Moreover, they [4] showed that if, in addition, $u_{n}$ is an energy-minimizing sequence in the same homotopy class, then the limit geodesics are all of finite length and they contain no energy. In fact, nontrivial geodesics arise only from the degeneration of conformal structures, not from "bubbling".

In order to understand how energy is lost when the domain surfaces degenerate, we shall give a precise expression of the energy loss near the nodes and then show the so-called generalized energy identity for harmonic maps from degenerating surfaces.

Let $u$ be a harmonic map defined on a standard cylinder $P=\left[t_{1}, t_{2}\right] \times S^{1}$ with flat metric $d s^{2}=d t^{2}+d \theta^{2}$. Then the Hopf quadratic differential of $u$ on $P$ is given by $\Phi(u)=$ $\phi(u)(d t+i d \theta)^{2}$, where

$$
\phi(u)=\left|u_{t}\right|^{2}-\left|u_{\theta}\right|^{2}-2 i u_{t} \cdot u_{\theta} .
$$

Our main observation is that the following integral:

$$
\int_{\{t\} \times S^{1}} \phi(u) d \theta
$$

is independent of $t \in\left[t_{1}, t_{2}\right]$. Thus (1.3) defines a complex number, which we denote by $\alpha=\alpha(u, P)$. We will see that this quantity can be applied to study the asymptotic behaviour of the necks appearing near the nodes (or punctures).

Now we consider a sequence of harmonic maps

$$
u_{n}:\left(\Sigma_{n}, h_{n}, c_{n}\right) \rightarrow N
$$

with uniformly bounded energy $E\left(u_{n}, \Sigma_{n}\right) \leq \Lambda<\infty$, where $\left(\Sigma_{n}, h_{n}, c_{n}\right)$ is a sequence of closed hyperbolic Riemann surfaces of genus $g>1$ with hyperbolic metrics $h_{n}$ and 
compatible complex structures $c_{n}$. Assume that $\left(\Sigma_{n}, h_{n}, c_{n}\right)$ degenerates to a hyperbolic Riemann surface $(\Sigma, h, c)$ by collapsing $p(1 \leq p \leq 3 g-3)$ pairwise disjoint simple closed geodesics $\gamma_{n}^{j}, j=1,2, \ldots, p$. For each $j$, the geodesics $\gamma_{n}^{j}$ degenerate into a pair of punctures $\left(\mathcal{E}^{j, 1}, \mathcal{E}^{j, 2}\right)$. Denote the $h_{n}$-length of $\gamma_{n}^{j}$ by $l_{n}^{j}$, and let $P_{n}^{j}$ be the standard cylindrical collar about $\gamma_{n}^{j}$.

We associate the sequence $\left(u_{n}, \Sigma_{n}\right)$ with a sequence of $p$-tuples $\left(\alpha_{n}^{1}, \ldots, \alpha_{n}^{p}\right)$, where $\alpha_{n}^{j}:=\alpha\left(u_{n}, P_{n}^{j}\right) \in \mathbb{C}$ are the quantities defined via (1.3). Pulling back the hyperbolic metrics $h_{n}$ and the compatible complex structures $c_{n}$ by suitable diffeomorphisms $\Sigma \rightarrow \Sigma_{n} \backslash \cup_{j=1}^{p} \gamma_{n}^{j}$ and passing to a subsequence, we can think of $\left(h_{n}, c_{n}\right)$ as all living on the limit surface $\Sigma$ and converging in $C_{l o c}^{\infty}$ to $(h, c)$. Thus, $u_{n}$ becomes a sequence of harmonic maps defined on $\left(\Sigma, h_{n}, c_{n}\right)$. Then we will show the following generalized energy identity for harmonic maps from degenerating surfaces:

Theorem 1.1 Let $u_{n}:\left(\Sigma_{n}, h_{n}, c_{n}\right) \rightarrow N$ be a sequence of harmonic maps with uniformly bounded energy $E\left(u_{n}, \Sigma_{n}\right) \leq \Lambda<\infty$, where $\left(\Sigma_{n}, h_{n}, c_{n}\right)$ is a sequence of closed hyperbolic Riemann surfaces of genus $g>1$ degenerating to a hyperbolic Riemann surface $(\Sigma, h, c)$ by collapsing finitely many pairwise disjoint simple closed geodesics $\left\{\gamma_{n}^{j}, j=1,2, \ldots, p\right\}$. Then, after selection of a subsequence, there exist finitely many blowup points $\left\{x_{1}, x_{2}, \ldots, x_{I}\right\}$ which are away from the punctures $\left\{\left(\mathcal{E}^{j, 1}, \mathcal{E}^{j, 2}\right), j=1,2, \ldots p\right\}$, and finitely many harmonic maps

$u:(\bar{\Sigma}, \bar{c}) \rightarrow N$, where $(\bar{\Sigma}, \bar{c})$ is the normalization of $(\Sigma, c)$, $\sigma^{i, l}: S^{2} \rightarrow N, l=1,2, \ldots, L_{i}$, near the ith blow up point $x_{i}$, $\omega^{j, k}: S^{2} \rightarrow N, k=1,2, \ldots, K_{j}$, near the $j$ th pair of punctures $\left(\mathcal{E}^{j, 1}, \mathcal{E}^{j, 2}\right)$, such that $u_{n}$ converges to $u$ in $C_{\text {loc }}^{\infty}$ on $\Sigma \backslash\left\{x_{i}, i=1,2, \ldots, I\right\}$ and the following holds

$$
\lim _{n \rightarrow \infty} E\left(u_{n}\right)=E(u)+\sum_{i=1}^{I} \sum_{l=1}^{L_{i}} E\left(\sigma^{i, l}\right)+\sum_{j=1}^{p} \sum_{k=1}^{K_{j}} E\left(\omega^{j, k}\right)+\sum_{j=1}^{p} \lim _{n \rightarrow \infty}\left|\operatorname{Re} \alpha_{n}^{j}\right| \cdot \frac{\pi^{2}}{l_{n}^{j}} .
$$

Moreover, for each $j$, there are at most finitely many necks connecting the base $u$ and the bubbles $\omega^{j, k}$. The sum of the average lengths (see Sect. 3) of those necks is asymptotically equal to

$$
\sqrt{\left|\operatorname{Re} \alpha_{n}^{j}\right|} \cdot \frac{\pi^{2}}{l_{n}^{j}}
$$

In fact, we have

Theorem 1.2 Assumptions and notations as in Theorem 1.1. Then

(1) $\left(u_{n}, \Sigma_{n}\right)$ subconverge in $W^{1,2}$ modulo bubbles, i.e., in the limit, the necks contain no energy if and only if

$$
\liminf _{n \rightarrow \infty}\left|\operatorname{Re} \alpha_{n}^{j}\right| \cdot \frac{\pi^{2}}{l_{n}^{j}}=0, \quad j=1,2, \ldots, p .
$$

(2) $\left(u_{n}, \Sigma_{n}\right)$ subconverge in $C^{0}$ modulo bubbles, i.e., in the limit, the images of the necks become points if and only if

$$
\liminf _{n \rightarrow \infty} \sqrt{\left|\operatorname{Re} \alpha_{n}^{j}\right|} \cdot \frac{\pi^{2}}{l_{n}^{j}}=0, \quad j=1,2, \ldots, p .
$$


It is clear from the above theorem that the limits $\liminf _{n \rightarrow \infty}\left|\operatorname{Re} \alpha_{n}^{j}\right| \cdot \frac{\pi^{2}}{l_{n}^{j}}, j=1,2, \ldots, p$ are the obstructions for $\left(u_{n}, \Sigma_{n}\right)$ to subconverge in $W^{1,2}$ modulo bubbles, and the limits $\liminf _{n \rightarrow \infty} \sqrt{\left|\operatorname{Re} \alpha_{n}^{j}\right|} \cdot \frac{\pi^{2}}{l_{n}^{j}}, j=1,2, \ldots, p$ are the obstructions for $\left(u_{n}, \Sigma_{n}\right)$ to subconverge in $C^{0}$ modulo bubbles. For each $j$, the asymptotic behaviour of the necks appearing near the $j$ th node is characterized by $\left\{\left(\alpha_{n}^{j}, l_{n}^{j}\right)\right\}_{n=1}^{\infty}$, namely

$$
E^{j} \approx\left|\operatorname{Re} \alpha_{n}^{j}\right| \cdot \frac{\pi^{2}}{l_{n}^{j}}, \quad L^{j} \approx \sqrt{\left|\operatorname{Re} \alpha_{n}^{j}\right|} \cdot \frac{\pi^{2}}{l_{n}^{j}},
$$

where $E^{j}$ is the sum of the energies of the necks and $L^{j}$ is the sum of the average lengths of the necks. Note that the quantities $\left\{\left(\alpha_{n}^{j}, l_{n}^{j}\right)\right\}_{n \geq 1}, j=1,2, \ldots, p$ are defined a priori.

For the asymptotics of the imaginary part of $\alpha_{n}^{j}$, we have the following:

\section{Proposition 1.1 Assumptions and notations as in Theorem 1.1. Then}

$$
\limsup _{n \rightarrow \infty}\left|\operatorname{Im} \alpha_{n}^{j}\right| \cdot \frac{\pi^{2}}{l_{n}^{j}}=0, \quad j=1,2, \ldots, p .
$$

When the domain surfaces of (1.1) are degenerating tori, then the study of the asymptotics of the necks is simpler because of the fact that any holomorphic quadratic differential on a torus is a constant. Some modifications to Parker's example [22] can illustrate the asymptotics mentioned, we refer to [40].

Wolf [36] studied the asymptotics of families of harmonic maps between hyperbolic surfaces where the domain degenerates via pinching finitely many pairwise disjoint simple closed geodesics. In this case, the energy of the maps goes to infinity. For the asymptotics of harmonic maps from surfaces to hyperbolic surfaces or hyperbolic three-manifolds, where the surfaces degenerate along a Teichmüller ray, see [19,20,35,37].

There are various energy identities for sequences of different approximations of harmonic maps from a fixed surface: for a min-max sequence by Jost [13]; for Struwe's harmonic map flow and certain Palais-Smale sequences with uniformly $L^{2}$-bounded tension field, see $[6,16,24,26,31,32,34]$; for minimizing sequences of Sacks-Uhlenbeck approximation of harmonic maps by Chen-Tian [4]; for the fourth order approximation of harmonic maps, see [17]. However, the energy identity for general sequences of Sacks-Uhlenbeck approximations is still open, a natural question then is whether a certain generalized energy identity holds. Based on the observations made by Qing [25] and Topping [33], one expects a complete understanding of the asymptotic behaviour of the necks appearing near the finite time singularity of the harmonic map flow. It would be interesting to ask whether one can associate to the flow suitable quantities that characterize the asymptotics of the necks mentioned above in a uniform way. If so, then a classification of the asymptotics in terms of these quantities is desirable. Recently, Ding et al. [7] introduced a flow for minimal tori and proved the corresponding energy identity. Considered as certain Palais-Smale sequences from degenerating tori, its higher genus generalization is expected.

Now, we briefly outline the remaining parts of the paper. In Sect. 2 we recall some preliminary facts about harmonic maps from surfaces. In Sect. 3 we develop several analytic properties of harmonic maps from long cylinders. In Sect. 4 we study harmonic maps from degenerating surfaces and prove Theorems 1.1 and 1.2. 


\section{Preliminaries}

Let $(\Sigma, h)$ be a Riemann surface with a metric $h=\lambda^{2} d z d \bar{z}$ in conformal coordinates $z=x+i y$. Let $(N, g)$ be a compact Riemannian manifold of dimension $d$, and let its metric in local coordinates be given by $g_{i j}$, with Christoffel symbols $\Gamma_{k l}^{i}$.

For $u \in W^{1,2}(\Sigma, N)$, the energy of $u$ on $\Sigma$ is

$$
E(u, \Sigma)=\frac{1}{2} \int_{\Sigma} g_{i j}\left(u_{x}^{i} u_{x}^{j}+u_{y}^{i} u_{y}^{j}\right) d x d y .
$$

A solution of the corresponding Euler-Lagrange equations

$$
\Delta u^{i}+\Gamma_{k l}^{i}\left(u_{x}^{k} u_{x}^{l}+u_{y}^{k} u_{y}^{l}\right)=0, \quad i=1, \ldots, d,
$$

is called a harmonic map. Note that (2.1) and (2.2) are conformally invariant.

If $u$ is in addition conformal, i.e., if the following holds:

$$
g_{j k}\left(u_{x}^{j} u_{x}^{k}-u_{y}^{j} u_{y}^{k}-2 i u_{x}^{j} u_{y}^{k}\right)=0,
$$

then $u$ is called a (parametric) minimal surface in $N$.

If we isometrically embed $N$ into some Euclidian space $\mathbb{R}^{K}$, then (2.2) can be written as follows:

$$
-\Delta u=A(u)(\nabla u, \nabla u),
$$

where $A(\cdot, \cdot)$ is the second fundamental form of $N$ in $\mathbb{R}^{K}$. Any $u \in W^{1,2}(\Sigma, N)$ that satisfies (2.3) weakly is smooth ([10,11], or [28] for a new proof).

For $u \in W^{1,2}(\Sigma, N)$, the Hopf quadratic differential associated to $u$ is defined by $\Phi(u)=$ $\phi(u) d z^{2}$, where

$$
\phi(u)=\left|u_{x}\right|^{2}-\left|u_{y}\right|^{2}-2 i u_{x} \cdot u_{y} .
$$

Lemma $2.1 u$ harmonic $\Rightarrow \phi(u)$ holomorphic. Also, $\phi(u) \equiv 0 \Leftrightarrow u$ is conformal.

For a proof, see for instance [13], Lemma 1.2.2.

We list some analytic facts about two-dimensional harmonic maps proved in [29].

Theorem 2.1 There exists a constant $\epsilon_{0}>0$ that depends only on $N$ such that

(1) ( $\epsilon$-regularity) Let $u: D \rightarrow N$ be a smooth harmonic map satisfying

$$
E(u, D)=\frac{1}{2} \int_{D}|d u|^{2} \leq \epsilon_{0} .
$$

Then

$$
\|d u\|_{\widetilde{D}, 1, p} \leq C(\widetilde{D}, p)\|d u\|_{D, 0,2},
$$

$\forall \widetilde{D} \subset \subset D$ and $p>1$, where $D$ is some regular domain in $\mathbb{R}^{2}, \widetilde{D}$ is any regular subdomain of $D$ and $C(\widetilde{D}, p)>1$ is a constant depending only on $\widetilde{D}, p$, and the geometry of $N$.

(2) (Singularity removability) Let $u$ be a smooth finite-energy harmonic map from a punctured disk $D \backslash\{0\}$ to $N$. Then u extends to a smooth harmonic map from $D$ to $N$.

(3) Any non-trivial harmonic map $u: S^{2} \rightarrow N$ has energy $E(u) \geq \epsilon_{0}$. 
Then, we have the following energy identity theorem $[13,22,29]$.

Theorem 2.2 Let $\left\{h_{n}\right\}$ be a sequence of Riemannian metrics on $\Sigma$ converging in $C^{\infty}$ to a Riemannian metric $h$, and let $u_{n}:\left(\Sigma, h_{n}\right) \rightarrow(N, g)$ be a sequence of $h_{n}$-harmonic maps with uniformly bounded energy $E\left(u_{n}\right) \leq \Lambda$. Then there are finitely many blow-up points $\left\{x_{1}, x_{2}, \ldots, x_{I}\right\} \subset \Sigma$, an h-harmonic map $u:(\Sigma, h) \rightarrow(N, g)$ and finitely many nontrivial harmonic maps $\sigma^{i, l}: S^{2} \rightarrow N, i=1,2, \ldots, I ; l=1,2, \ldots, L_{i}$, such that after selection of a subsequence, $u_{n}$ converges in $C_{\text {loc }}^{\infty}$ to $u$ on $\Sigma \backslash\left\{x_{1}, x_{2}, \ldots, x_{I}\right\}$, and the following holds

$$
\lim _{n \rightarrow \infty} E\left(u_{n}\right)=E(u)+\sum_{i=1}^{I} \sum_{l=1}^{L_{i}} E\left(\sigma^{i, l}\right) .
$$

During the blow-up process, some necks connecting one bubble to the next or connecting the base to a bubble appear. Theorem 2.2 shows that in the limit those necks contain no energy. In this case, we say $u_{n}$ subconverges to $u$ in $W^{1,2}$ modulo bubbles. Moreover, Parker [22] proved that all necks converge to points in the target manifold, i.e., $u_{n}$ subconverges to $u$ in $C^{0}$ modulo bubbles. Using our terminology, we simply state Parker's results as follows:

Theorem 2.3 (Bubble tree convergence) Notations and assumptions as in Theorem 2.2. Then, after selection of a subsequence, $u_{n}$ converges to $u$ in $W^{1,2} \cap C^{0}$ modulo bubbles.

For more details on the construction of the bubble trees, see [22,23].

\section{Harmonic maps from cylinders}

In this section, we study harmonic maps from cylinders and derive some analytic properties.

Let $P_{T_{1}, T_{2}}=\left[T_{1}, T_{2}\right] \times S^{1}$ be a standard cylinder with metric $d s^{2}=d t^{2}+d \theta^{2}$, here $S^{1}=\mathbb{R} / 2 \pi \mathbb{Z}$. Since we will only need to consider long cylinders, w.l.o.g., we always assume that $T_{2}-T_{1}>2$. Let $u: P_{T_{1}, T_{2}} \rightarrow N$ be a $C^{1}$ map. Denote

$$
\Theta(t):=\int_{\{t\} \times S^{1}}\left|u_{\theta}\right|^{2} .
$$

The following lemma is a modified version of two lemmas proved in [16,22]. For the reader's convenience, we will give a proof using arguments from [16].

Lemma 3.1 There exists $\epsilon_{1}>0$, only depending on $N$, such that if $u: P_{T_{1}, T_{2}} \rightarrow N$ is a harmonic map and

$$
\sup _{P_{T_{1}, T_{2}}}|\nabla u| \leq \epsilon_{1},
$$

then

$$
\frac{d^{2}}{d t^{2}} \Theta(t) \geq \Theta(t), \quad \forall t \in\left[T_{1}, T_{2}\right]
$$

Moreover, we have

$$
\int_{T_{1}}^{T_{2}}(\Theta(t))^{v} d t \leq 2 \frac{\left(\left(\Theta\left(T_{1}\right)\right)^{v}+\left(\Theta\left(T_{2}\right)\right)^{v}\right)}{v}, \quad \forall v \in(0,1] .
$$


Proof By a straightforward calculation as in [16], Lemma 2.1, we have

$$
\frac{d^{2}}{d t^{2}} \int_{S^{1}}\left|u_{\theta}\right|^{2} \geq\left(2-C \epsilon_{1}^{2}\right) \int_{S^{1}}\left|u_{\theta t}\right|^{2}+\left(\frac{3}{2}-C \epsilon_{1}^{2}\right) \int_{S^{1}}\left|u_{\theta \theta}\right|^{2}-\epsilon_{1}^{2} \int_{S^{1}}\left|u_{\theta}\right|^{2} .
$$

Here $C$ is a constant depending only on the geometry of $N$. If we choose $\epsilon_{1}>0$ small enough, then

$$
\frac{d^{2}}{d t^{2}} \int_{S^{1}}\left|u_{\theta}\right|^{2} \geq \frac{5}{4} \int_{S^{1}}\left|u_{\theta \theta}\right|^{2}-\frac{1}{4} \int_{S^{1}}\left|u_{\theta}\right|^{2} \geq \int_{S^{1}}\left|u_{\theta}\right|^{2} .
$$

Here, in the last step, we used the Poincaré inequality on $S^{1}$. This proves (3.1).

Let $\tau_{i}=\Theta\left(T_{i}\right), i=1,2$. Then we can solve the following 2 nd order ODE:

$$
\begin{aligned}
& \ddot{\rho}-\rho=0, \quad T_{1} \leq t \leq T_{2}, \\
& \rho\left(T_{1}\right)=\tau_{1}, \\
& \rho\left(T_{2}\right)=\tau_{2} .
\end{aligned}
$$

and obtain a solution $\rho(t)=\lambda e^{t}+\mu e^{-t}$, where

$$
\lambda=\frac{\left(e^{T_{2}} \tau_{2}-e^{T_{1}} \tau_{1}\right)}{e^{2 T_{2}}-e^{2 T_{1}}}, \quad \mu=\frac{e^{T_{1}+2 T_{2}} \tau_{2}-e^{2 T_{1}+T_{2}} \tau_{1}}{e^{2 T_{2}}-e^{2 T_{1}}} .
$$

Applying the maximum principle, we conclude

$$
0 \leq \Theta(t) \leq \rho(t), \quad \forall t \in\left[T_{1}, T_{2}\right] .
$$

Note that $T_{2}>T_{1}, \tau_{1} \geq 0, \tau_{2} \geq 0$, and $v \in(0,1]$. By direct calculation, we have

$$
\begin{aligned}
\int_{T_{1}}^{T_{2}}(\Theta(t))^{v} d t & \leq|\lambda|^{\nu} \frac{\left(e^{\nu T_{2}}-e^{\nu T_{1}}\right)}{v}+|\mu|^{\nu} \frac{\left(e^{-\nu T_{1}}-e^{-\nu T_{2}}\right)}{v} \\
& \leq 2 \frac{\left|e^{T_{2}} \tau_{2}-e^{T_{1}} \tau_{1}\right|^{v}}{\left(e^{2 T_{2}}-e^{2 T_{1}}\right)^{v}} \cdot \frac{\left(e^{\nu T_{2}}-e^{\nu T_{1}}\right)}{v} \\
& \leq 2 \frac{\left.\left(\left(\tau_{1}\right)\right)^{v}+\left(\tau_{2}\right)^{v}\right)}{v} \\
& =2 \frac{\left(\left(\Theta\left(T_{1}\right)\right)^{v}+\left(\Theta\left(T_{2}\right)\right)^{v}\right)}{v} .
\end{aligned}
$$

This gives (3.2). We have thus finished the proof.

Combining Lemma 3.1 and the " $\epsilon$-regularity" portion of Theorem 2.1, we have

Lemma 3.2 There exist $\epsilon_{2}>0$ and $C>0$, depending only on $N$, such that if $u$ is a harmonic map from $P_{T_{1}, T_{2}}$ to $N$ and

$$
\omega:=\sup _{t \in\left[T_{1}, T_{2}-1\right]} \int_{[t, t+1] \times S^{1}}|d u|^{2} \leq \epsilon_{2},
$$

then

$$
\int_{T_{1}}^{T_{2}} \Theta(t) d t \leq C \omega, \quad \int_{T_{1}}^{T_{2}} \sqrt{\Theta(t)} d t \leq C \omega^{\frac{1}{2}}
$$


Proof Let $\epsilon_{0}>0$ be the constant in Theorem 2.1 (" $\epsilon$-regularity"), and let $\epsilon_{1}>0$ be the constant in Lemma 3.1. Let $\epsilon_{2}=\min \left\{\epsilon_{0},\left(\frac{\epsilon_{1}}{C_{0}}\right)^{2}\right\}$, where $C_{0}$ is a constant to be determined later. If

$$
\omega=\sup _{t \in\left[T_{1}, T_{2}-1\right]} \int_{[t, t+1] \times S^{1}}|d u|^{2} \leq \epsilon_{2},
$$

then changing variables by translating in $u$ and using $\epsilon$-regularity property with $D=$ $[-1,2] \times S^{1}$ and $\widetilde{D}=[0,1] \times S^{1}$, we have

$$
\sup _{\left[T_{1}+1, T_{2}-1\right] \times S^{1}}|\nabla u| \leq C_{1}\left(\sup _{t \in\left[T_{1}, T_{2}-1\right]} \int_{[t, t+1] \times S^{1}}|d u|^{2}\right)^{\frac{1}{2}}=C_{1} \omega^{\frac{1}{2}} \leq \frac{C_{1}}{C_{0}} \epsilon_{1} .
$$

where $C_{1}>0$ is a constant depending only on $N$, but not on $T_{1}, T_{2}$. We take $C_{0}$ to be the constant $C_{1}$ here. Then we can apply Lemma 3.1 to conclude that $\forall v \in(0,1]$,

$$
\int_{T_{1}+1}^{T_{2}-1}(\Theta(t))^{v} d t \leq 2 \frac{\left(\left(\Theta\left(T_{1}+1\right)\right)^{v}+\left(\Theta\left(T_{2}-1\right)\right)^{v}\right)}{v} \leq C \sup _{P_{T_{1}+1, T_{2}-1}}|\nabla u|^{2 v} \leq C \omega^{v} .
$$

On the other hand, it is not hard to verify that (applying Hölder's inequality if necessary)

$$
\int_{T_{1}}^{T_{1}+1}(\Theta(t))^{v} d t+\int_{T_{2}-1}^{T_{2}}(\Theta(t))^{v} d t \leq 2 \omega^{v}, \quad \forall v \in(0,1] .
$$

(3.3) follows from combining (3.4) and (3.5) and taking $v=1, \frac{1}{2}$.

Lemma 3.3 Let $u: P_{T_{1}, T_{2}} \rightarrow N$ be a harmonic map. Then for $t \in\left[T_{1}, T_{2}\right]$,

$$
\int_{\{t\} \times S^{1}} \phi(u) d \theta
$$

is independent of $t \in\left[T_{1}, T_{2}\right]$, where

$$
\phi(u)=\left|u_{t}\right|^{2}-\left|u_{\theta}\right|^{2}-2 i u_{t} \cdot u_{\theta}
$$

and $\phi(u)(d t+i d \theta)^{2}$ is the Hopf quadratic differential of $u$ on $P_{T_{1}, T_{2}}$.

Proof By Lemma 2.1, we know that if $u$ is harmonic then $\phi(u)$ is holomorphic. Given $t_{1}$ and $t_{2}$ such that $T_{1} \leq t_{1} \leq t_{2} \leq T_{2}$, consider the rectangle $R$ bounded by $\left[t_{1}, t_{2}\right] \times\{0\},\left\{t_{2}\right\} \times$ $[0,2 \pi],\left[t_{2}, t_{1}\right] \times\{2 \pi\}$, and $\left\{t_{1}\right\} \times[2 \pi, 0]$. By Cauchy's integral theorem, we have

$$
\oint_{\partial R} \phi(u)=\int_{R} \bar{\partial} \phi(u)=0,
$$

i.e.,

$$
\int_{\left\{t_{1}\right\} \times S^{1}} \phi(u) d \theta=\int_{\left\{t_{2}\right\} \times S^{1}} \phi(u) d \theta .
$$

Hence (3.6) is independent of $t \in\left[T_{1}, T_{2}\right]$. 
Definition 3.1 Let $u: P_{T_{1}, T_{2}} \rightarrow N$ be harmonic. Then we define a complex number

$$
\alpha\left(u, P_{T_{1}, T_{2}}\right):=\int_{\{t\} \times S^{1}} \phi(u) d \theta \in \mathbb{C}
$$

that is associated to $u$ along the cylinder $P_{T_{1}, T_{2}}$.

Remark 3.1 It follows from Lemma 3.3 that $\alpha\left(u, P_{T_{1}, T_{2}}\right)$ is well-defined. Moreover, we have $\alpha\left(u, P_{t_{1}^{\prime}, t_{2}^{\prime}}\right)=\alpha\left(u, P_{t_{1}, t_{2}}\right), \forall t_{1}<t_{1}^{\prime}<t_{2}^{\prime}<t_{2}$.

Definition 3.2 Let $u: P_{T_{1}, T_{2}} \rightarrow N$ be a $C^{1}$ map. Then we call

$$
L\left(u, P_{T_{1}, T_{2}}\right):=\int_{T_{1}}^{T_{2}}\left(\int_{0}^{2 \pi}\left|u_{t}\right|^{2} d \theta\right)^{\frac{1}{2}} d t .
$$

the average length of $u$ along the cylinder $P_{T_{1}, T_{2}}$.

Remark 3.2 Let $c:\left[T_{1}, T_{2}\right] \rightarrow N$ be a $C^{1}$ curve in $N$. Then $u(t, \theta):=c(t)$ is a $\theta$-independent $C^{1}$ map from $P_{T_{1}, T_{2}}$ to $N$. It is easy to verify that

$$
L\left(u, P_{T_{1}, T_{2}}\right)=\sqrt{2 \pi} L\left(c,\left[T_{1}, T_{2}\right]\right),
$$

where $L\left(c,\left[T_{1}, T_{2}\right]\right)=\int_{T_{1}}^{T_{2}}|\dot{c}(t)| d t$ is the usual length of the curve $c$.

Lemma 3.4 Let $u: P_{T_{1}, T_{2}} \rightarrow N$ be a harmonic map with $\alpha=\alpha\left(u, P_{T_{1}, T_{2}}\right)$. Then we have (1)

$$
\left|E\left(u, P_{T_{1}, T_{2}}\right)-\frac{1}{2}\right| \operatorname{Re} \alpha\left|\cdot\left(T_{2}-T_{1}\right)\right| \leq \int_{T_{1}}^{T_{2}} \Theta(t) d t,
$$

(2)

$$
\left|L\left(u, P_{T_{1}, T_{2}}\right)-\sqrt{|\operatorname{Re} \alpha|} \cdot\left(T_{2}-T_{1}\right)\right| \leq \int_{T_{1}}^{T_{2}} \sqrt{\Theta(t)} d t,
$$

(3)

$$
|\operatorname{Im} \alpha| \cdot\left(T_{2}-T_{1}\right) \leq 2 \sqrt{2 E\left(u, P_{T_{1}, T_{2}}\right)} \cdot \sqrt{\int_{T_{1}}^{T_{2}} \Theta(t) d t .}
$$

Proof In view of Definition 3.1, we have

$$
\operatorname{Re} \alpha=\int_{0}^{2 \pi}\left|u_{t}\right|^{2} d \theta-\int_{0}^{2 \pi}\left|u_{\theta}\right|^{2} d \theta, \quad \operatorname{Im} \alpha=-2 \int_{0}^{2 \pi} u_{t} \cdot u_{\theta} d \theta .
$$

Then by applying the following inequalities,

$$
|(a+b)-| a|| \leq b, \quad|\sqrt{a+b}-\sqrt{|a|}| \leq \sqrt{b}, \quad \forall a, b, a+b \geq 0, b \geq 0,
$$

and then integrating with respect to $t$, we get (3.8) and (3.9). Equation (3.10) follows from the Cauchy inequality. 
The next lemma is inspired by [22].

Lemma 3.5 Let $u: P_{T_{1}, T_{2}} \rightarrow N$ be a $C^{1}$ map. Then

$$
\underset{P_{T_{1}, T_{2}}}{\operatorname{osc}} u \leq 4 \pi \sup _{P_{T_{1}, T_{2}}}|\nabla u|+\frac{1}{\sqrt{2 \pi}} L\left(u, P_{T_{1}, T_{2}}\right) .
$$

Proof Let $\left(t_{1}, \theta_{1}\right),\left(t_{2}, \theta_{2}\right) \in P_{T_{1}, T_{2}}=\left[T_{1}, T_{2}\right] \times S^{1}$, where $T_{1} \leq t_{1}<t_{2} \leq T_{2}$. Then by the Mean Value Theorem for integration, there exists $\theta_{0} \in[0,2 \pi]$ such that

$$
\int_{t_{1}}^{t_{2}}\left|u_{t}\left(t, \theta_{0}\right)\right| d t=\frac{1}{2 \pi} \int_{0}^{2 \pi} \int_{t_{1}}^{t_{2}}\left|u_{t}\right| d t d \theta
$$

Hence, we have

$$
\begin{aligned}
\operatorname{dist}\left(u\left(t_{1}, \theta_{1}\right), u\left(t_{2}, \theta_{2}\right)\right) \leq & \operatorname{dist}\left(u\left(t_{1}, \theta_{1}\right), u\left(t_{1}, \theta_{0}\right)\right)+\operatorname{dist}\left(u\left(t_{1}, \theta_{0}\right), u\left(t_{2}, \theta_{0}\right)\right) \\
& +\operatorname{dist}\left(u\left(t_{2}, \theta_{0}\right), u\left(t_{2}, \theta_{2}\right)\right) \\
= & I+I I+I I I,
\end{aligned}
$$

It is easy to see that $I+I I I \leq \int_{0}^{2 \pi}\left|u_{\theta}\left(t_{1}, \theta\right)\right| d \theta+\int_{0}^{2 \pi}\left|u_{\theta}\left(t_{2}, \theta\right)\right| d \theta \leq 4 \pi \cdot \sup _{P_{T_{1}, T_{2}}}|\nabla u|$. By (3.12) and the Cauchy inequality, we conclude

$$
I I \leq \int_{t_{1}}^{t_{2}}\left|u_{t}\left(t, \theta_{0}\right)\right| d t=\frac{1}{2 \pi} \cdot \int_{0}^{2 \pi} \int_{t_{1}}^{t_{2}}\left|u_{t}\right| d t d \theta \leq \frac{1}{\sqrt{2 \pi}} \cdot L\left(u, P_{T_{1}, T_{2}}\right) .
$$

(3.11) follows immediately.

Based on the neck analysis in [6], we have the following proposition, which gives a refined "bubble domain and neck domain" decomposition for a sequence of harmonic maps from long cylinders under certain assumptions.

Proposition 3.1 Let $u_{n} \in C^{\infty}\left(P_{n}, N\right)$ be a sequence of harmonic maps with $\alpha_{n}=\alpha\left(u_{n}, P_{n}\right)$, where $P_{n}=\left[T_{n}^{1}, T_{n}^{2}\right] \times S^{1}$. Assume that:

(1) "Long cylinder property"

$$
1 \ll T_{n}^{1} \ll T_{n}^{2}, \text { i.e., } \lim _{n \rightarrow \infty} \frac{1}{T_{n}^{1}}=0, \lim _{n \rightarrow \infty} \frac{T_{n}^{1}}{T_{n}^{2}}=0,
$$

(2) "Uniform energy bound"

$$
E\left(u_{n}, P_{n}\right) \leq \Lambda<\infty,
$$

(3) "Asymptotic boundary conditions"

$$
\begin{aligned}
& \lim _{n \rightarrow \infty} \omega\left(u_{n}, P_{T_{n}^{1}, T_{n}^{1}+R}\right)=\lim _{n \rightarrow \infty} \omega\left(u_{n}, P_{T_{n}^{2}-R, T_{n}^{2}}\right)=0, \quad \forall R \geq 1,
\end{aligned}
$$

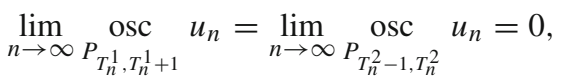

where

$$
\omega\left(u, P_{T_{1}, T_{2}}\right):=\sup _{t \in\left[T_{1}, T_{2}-1\right]} \int_{[t, t+1] \times S^{1}}|d u|^{2} .
$$


Then, after selection of a subsequence, which we still denote by $\left(u_{n}, P_{n}\right)$, either

(I)

$$
\lim _{n \rightarrow \infty} \omega\left(u_{n}, P_{n}\right)=0
$$

or

(II) $\exists K>0$ independent of $n$ and $2 K$ sequences $\left\{a_{n}^{1}\right\},\left\{b_{n}^{1}\right\},\left\{a_{n}^{2}\right\},\left\{b_{n}^{2}\right\}, \ldots,\left\{a_{n}^{K}\right\},\left\{b_{n}^{K}\right\}$ such that

$$
\left.T_{n}^{1} \leq a_{n}^{1} \ll b_{n}^{1} \leq \cdots \leq a_{n}^{K} \ll b_{n}^{K} \leq T_{n}^{2} \text { (here } a_{n}^{i} \ll b_{n}^{i} \text { means } \lim _{n \rightarrow \infty} b_{n}^{i}-a_{n}^{i}=\infty\right)
$$

and

$$
\left(b_{n}^{i}-a_{n}^{i}\right) \ll T_{n}^{2}, \text { i.e., } \lim _{n \rightarrow \infty} \frac{b_{n}^{i}-a_{n}^{i}}{T_{n}^{2}}=0, \quad i=1,2, \ldots, K .
$$

Denote

$$
\begin{aligned}
J_{n}^{j}:= & {\left[a_{n}^{j}, b_{n}^{j}\right] \times S^{1}, \quad j=1,2, \ldots, K, } \\
I_{n}^{0}:= & {\left[T_{n}^{1}, a_{n}^{1}\right] \times S^{1}, I_{n}^{K}:=\left[b_{n}^{K}, T_{n}^{2}\right] \times S^{1}, I_{n}^{i}:=\left[b_{n}^{i}, a_{n}^{i+1}\right] \times S^{1}, } \\
& i=1,2, \ldots, K-1 .
\end{aligned}
$$

Then

(i) $\forall i=0,1, \ldots, K, \lim _{n \rightarrow \infty} \omega\left(u_{n}, I_{n}^{i}\right)=0$. The maps $u_{n}: I_{n}^{i} \rightarrow N$ are necks corresponding to collapsing homotopically nontrivial curves.

(ii) $\forall j=1,2, \ldots, K$, there are finitely many harmonic maps $\omega^{j, l}: S^{2} \rightarrow N, l=$ $1,2, \ldots, L_{j}$, such that:

$$
\lim _{n \rightarrow \infty} E\left(u_{n}, J_{n}^{j}\right)=\sum_{l=1}^{L_{j}} E\left(\omega^{j, l}\right) .
$$

Proof If $\liminf _{n \rightarrow \infty} \omega\left(u_{n}, P_{n}\right)=0$, then, after selection of a subsequence, we get (3.16). Otherwise, w.l.o.g., we can assume that

$$
\lim _{n \rightarrow \infty} \omega\left(u_{n}, P_{n}\right)=\lim _{n \rightarrow \infty} \sup _{t \in\left[T_{n}^{1}, T_{n}^{2}-1\right]} \int_{[t, t+1] \times S^{1}}\left|d u_{n}\right|^{2} d t d \theta>0 .
$$

Then $\exists \epsilon>0,\left\{t_{n}\right\} \in\left[T_{n}^{1}, T_{n}^{2}-1\right]$, such that for all $n$ large enough,

$$
\int_{\left[t_{n}, t_{n}+1\right] \times S^{1}}\left|d u_{n}\right|^{2} d t d \theta \geq \epsilon .
$$

It follows from the "asymptotic boundary conditions" (3.15) that $t_{n}-T_{n}^{1} \rightarrow \infty$ and $T_{n}^{2}-t_{n} \rightarrow$ $\infty$. By translation $t \rightarrow t-t_{n}$, we can think of $u_{n}$ as a harmonic map defined on $\left[-R_{n}, R_{n}\right] \times S^{1}$ with $R_{n} \rightarrow \infty$ and

$$
\int_{[0,1] \times S^{1}}\left|d u_{n}\right|^{2} d t d \theta \geq \epsilon, \quad \int_{\left[-R_{n}, R_{n}\right] \times S^{1}}\left|d u_{n}\right|^{2} d t d \theta \leq \Lambda .
$$


As $n \rightarrow \infty,\left[-R_{n}, R_{n}\right] \times S^{1}$ exhaust $(-\infty, \infty) \times S^{1}$, which is conformally equivalent to $S^{2}$ with two punctures. Hence by the conformal invariance of two-dimensional harmonic maps and the bubble tree convergence theorem, we can choose a subsequence of $\left(u_{n}, P_{n}\right)$ (still denoted by $\left.\left(u_{n}, P_{n}\right)\right)$ such that there exist $\left\{a_{n}^{1}\right\},\left\{b_{n}^{1}\right\}$ satisfying

$$
T_{n}^{1} \leq a_{n}^{1} \ll b_{n}^{1} \leq T_{n}^{2}, \quad\left(b_{n}^{1}-a_{n}^{1}\right) \ll T_{n}^{2}
$$

and such that the following sequence of harmonic maps

$$
\widetilde{u}_{n}^{1}(t, \theta):\left[-\frac{b_{n}^{1}-a_{n}^{1}}{2}, \frac{b_{n}^{1}-a_{n}^{1}}{2}\right] \times S^{1} \rightarrow N
$$

converges to a bubble tree $\widetilde{u}_{\infty}^{1}$ (cf. [22]), where $\widetilde{u}_{n}^{1}(t, \theta):=u_{n}\left(t+\frac{a_{n}^{1}+b_{n}^{1}}{2}, \theta\right)$. Moreover, there exist finitely many harmonic maps $\omega^{1, l}: S^{2} \rightarrow N, l=1,2, \ldots, L_{1}$, such that

$$
\lim _{n \rightarrow \infty} E\left(u_{n},\left[a_{n}^{1}, b_{n}^{1}\right] \times S^{1}\right)=\lim _{n \rightarrow \infty} E\left(\widetilde{u}_{n}^{1}\right)=\sum_{l=1}^{L_{1}} E\left(\omega^{1, l}\right) .
$$

By (3.19), (3.20) and Theorem 2.1, we have $\limsup _{n \rightarrow \infty} E\left(u_{n}, P_{n} \backslash\left(\left[a_{n}^{1}, b_{n}^{1}\right] \times S^{1}\right)\right) \leq \Lambda-\epsilon_{0}$. Denote

$$
J_{n}^{1}:=\left[a_{n}^{1}, b_{n}^{1}\right] \times S^{1}, \quad I_{n}^{0}:=\left[T_{n}^{1}, a_{n}^{1}\right] \times S^{1}, \quad I_{n}^{1}:=\left[b_{n}^{1}, T_{n}^{2}\right] \times S^{1} .
$$

Then (3.20) becomes $\lim _{n \rightarrow \infty} E\left(u_{n}, J_{n}^{1}\right)=\sum_{l=1}^{L_{1}} E\left(\omega^{1, l}\right)$. After selection of a subsequence, $u_{n}: I_{n}^{i} \rightarrow N, i=0,1$, satisfy the conditions (3.14) and (3.15) with $\Lambda$ replaced by $\Lambda-\epsilon_{0}$. Now if the following hold:

$$
\liminf _{n \rightarrow \infty} \omega\left(u_{n}, I_{n}^{i}\right)=0, \quad i=0,1,
$$

then, after passing to a further subsequence, we finish the proof. Otherwise, we can do the same procedure as in the beginning of the proof [note that we do not need (3.13)] and take subsequences if necessary. The whole procedure ends within finitely many steps because of the uniform energy bound (3.14). The proof can be completed by induction on $K$, the number of the bubble trees, and reordering $\left\{a_{n}^{i}, b_{n}^{i}\right\}, i=1,2, \ldots, K$.

Now we study the limit of the energy and average lengths of the necks

$$
u_{n}: I_{n}^{i} \rightarrow N, \quad i=0,1, \ldots, K \text {. }
$$

Recall that these necks satisfy $\lim _{n \rightarrow \infty} \omega\left(u_{n}, I_{n}^{i}\right)=0$, hence we can apply Lemma 3.2 and Lemma 3.4 to estimate $E\left(u_{n}, I_{n}^{i}\right)$ and $L\left(u_{n}, I_{n}^{i}\right)$.

Main Proposition 3.1 Assumptions and notations as in Proposition 3.1, w.l.o.g., we assume that both $\lim _{n \rightarrow \infty}\left|\operatorname{Re} \alpha_{n}\right| \cdot\left|P_{n}\right|$ and $\lim _{n \rightarrow \infty} \sqrt{\left|\operatorname{Re} \alpha_{n}\right|} \cdot\left|P_{n}\right|$ exist in $[0,+\infty]$. Then

$$
\begin{aligned}
& \lim _{n \rightarrow \infty} \sum_{i=0}^{K} E\left(u_{n}, I_{n}^{i}\right)=\frac{1}{2} \lim _{n \rightarrow \infty}\left|\operatorname{Re} \alpha_{n}\right| \cdot\left|P_{n}\right|, \\
& \lim _{n \rightarrow \infty} \sum_{i=0}^{K} L\left(u_{n}, I_{n}^{i}\right)=\lim _{n \rightarrow \infty} \sqrt{\left|\operatorname{Re} \alpha_{n}\right|} \cdot\left|P_{n}\right| .
\end{aligned}
$$


Proof We write

$$
\begin{aligned}
\sum_{i=0}^{K} E\left(u_{n}, I_{n}^{i}\right) & =\sum_{i=0}^{K} \frac{1}{2}\left|\operatorname{Re} \alpha_{n}\right| \cdot\left|I_{n}^{i}\right|+\sum_{i=0}^{K}\left(E\left(u_{n}, I_{n}^{i}\right)-\frac{1}{2}\left|\operatorname{Re} \alpha_{n}\right| \cdot\left|I_{n}^{i}\right|\right) \\
& =I+I I,
\end{aligned}
$$

where

$$
\begin{aligned}
I & :=\sum_{i=0}^{K} \frac{1}{2}\left|\operatorname{Re} \alpha_{n}\right| \cdot\left|I_{n}^{i}\right| \\
& =\frac{1}{2}\left|\operatorname{Re} \alpha_{n}\right| \cdot\left[\left(T_{n}^{2}-T_{n}^{1}\right)-\sum_{i=1}^{K}\left(b_{n}^{i}-a_{n}^{i}\right)\right] \\
& =\frac{1}{2}\left|\operatorname{Re} \alpha_{n}\right| \cdot\left(T_{n}^{2}-T_{n}^{1}\right) \cdot\left(\frac{T_{n}^{2}}{T_{n}^{2}-T_{n}^{1}}\right) \cdot\left[\left(1-\frac{T_{n}^{1}}{T_{n}^{2}}\right)-\sum_{i=1}^{K} \frac{\left(b_{n}^{i}-a_{n}^{i}\right)}{T_{n}^{2}}\right]
\end{aligned}
$$

and

$$
I I:=\sum_{i=0}^{K}\left(E\left(u_{n}, I_{n}^{i}\right)-\frac{1}{2}\left|\operatorname{Re} \alpha_{n}\right| \cdot\left|I_{n}^{i}\right|\right) .
$$

Denote $\Theta_{n}(t)=\int_{\{t\} \times S^{1}}\left|\left(u_{n}\right)_{\theta}\right|^{2}$. By Lemma 3.2, Lemma 3.4 and Proposition 3.1,

$$
\begin{aligned}
|I I| & \leq \sum_{i=0}^{K}\left|E\left(u_{n}, I_{n}^{i}\right)-\frac{1}{2}\right| \operatorname{Re} \alpha_{n}|\cdot| I_{n}^{i}|| \\
& \leq \int_{T_{n}^{1}}^{a_{n}^{1}} \Theta_{n}(t) d t+\sum_{i=1}^{K-1} \int_{b_{n}^{i}}^{a_{n}^{i+1}} \Theta_{n}(t) d t+\int_{b_{n}^{K}}^{T_{n}^{2}} \Theta_{n}(t) d t \\
& \leq C(\Lambda) \sum_{i=0}^{K} \omega\left(u_{n}, I_{n}^{i}\right) \rightarrow 0, \quad n \rightarrow \infty
\end{aligned}
$$

We write

$$
\begin{aligned}
\sum_{i=0}^{K} L\left(u_{n}, I_{n}^{i}\right) & =\sum_{i=0}^{K} \sqrt{\left|\operatorname{Re} \alpha_{n}\right|} \cdot\left|I_{n}^{i}\right|+\sum_{i=0}^{K}\left(L\left(u_{n}, I_{n}^{i}\right)-\sqrt{\left|\operatorname{Re} \alpha_{n}\right|} \cdot\left|I_{n}^{i}\right|\right) \\
& =I I I+I V
\end{aligned}
$$

where

$$
\begin{aligned}
I I I & :=\sum_{i=0}^{K} \sqrt{\left|\operatorname{Re} \alpha_{n}\right|} \cdot\left|I_{n}^{i}\right| \\
& =\sqrt{\left|\operatorname{Re} \alpha_{n}\right|} \cdot\left[\left(T_{n}^{2}-T_{n}^{1}\right)-\sum_{i=1}^{K}\left(b_{n}^{i}-a_{n}^{i}\right)\right] \\
& =\sqrt{\left|\operatorname{Re} \alpha_{n}\right|} \cdot\left(T_{n}^{2}-T_{n}^{1}\right) \cdot\left(\frac{T_{n}^{2}}{T_{n}^{2}-T_{n}^{1}}\right) \cdot\left[\left(1-\frac{T_{n}^{1}}{T_{n}^{2}}\right)-\sum_{i=1}^{K} \frac{\left(b_{n}^{i}-a_{n}^{i}\right)}{T_{n}^{2}}\right]
\end{aligned}
$$


and

$$
I V:=\sum_{i=0}^{K}\left(L\left(u_{n}, I_{n}^{i}\right)-\sqrt{\left|\operatorname{Re} \alpha_{n}\right|} \cdot\left|I_{n}^{i}\right|\right) .
$$

Applying Lemmas 3.2, 3.4 and Proposition 3.1, we get

$$
\begin{aligned}
|I V| & =\sum_{i=0}^{K}\left|L\left(u_{n}, I_{n}^{i}\right)-\sqrt{\left|\operatorname{Re} \alpha_{n}\right|} \cdot\right| I_{n}^{i}|| \\
& \leq \int_{T_{n}^{1}}^{a_{n}^{1}} \sqrt{\Theta_{n}(t)} d t+\sum_{i=1}^{K-1} \int_{b_{n}^{i}}^{a_{n}^{i+1}} \sqrt{\Theta_{n}(t)} d t+\int_{b_{n}^{K}}^{T_{n}^{2}} \sqrt{\Theta_{n}(t)} d t \\
& \leq C(\Lambda) \sum_{i=0}^{K} \sqrt{\omega\left(u_{n}, I_{n}^{i}\right)} \rightarrow 0, \quad n \rightarrow \infty .
\end{aligned}
$$

Recall the properties (3.13), (3.17) and (3.18) in Proposition 3.1, namely

$$
1 \ll T_{n}^{1} \ll T_{n}^{2}, \quad 1 \ll\left(b_{n}^{i}-a_{n}^{i}\right) \ll T_{n}^{2}, \quad i=1,2, \ldots, K .
$$

Then, combining (3.23), (3.24) and (3.25), we conclude

$$
\lim _{n \rightarrow \infty} \sum_{i=0}^{K} E\left(u_{n}, I_{n}^{i}\right)=\lim _{n \rightarrow \infty}(I+I I)=\frac{1}{2} \lim _{n \rightarrow \infty}\left|\operatorname{Re} \alpha_{n}\right| \cdot\left|P_{n}\right| .
$$

Similarly, combining (3.26)-(3.28) gives

$$
\lim _{n \rightarrow \infty} \sum_{i=0}^{K} L\left(u_{n}, I_{n}^{i}\right)=\lim _{n \rightarrow \infty}(I I I+I V)=\lim _{n \rightarrow \infty} \sqrt{\left|\operatorname{Re} \alpha_{n}\right|} \cdot\left|P_{n}\right| .
$$

Thus we have proved (3.21) and (3.22).

Remark 3.3 It follows from Remark 3.1 that

$$
\alpha\left(u_{n}, I_{n}^{i}\right)=\alpha\left(u_{n}, P_{n}\right), \quad i=0,1, \ldots, K .
$$

Thus, we can study the properties of the necks $u_{n}: I_{n}^{i} \rightarrow N$ in a uniform way, but not separately.

Applying similar arguments as in the proof of Main Proposition 3.1, we get

Proposition 3.2 With the same assumptions and notations as in Proposition 3.1, we have

$$
\limsup _{n \rightarrow \infty}\left|\operatorname{Re} \alpha_{n}\right| \cdot\left|P_{n}\right| \leq 2 \Lambda, \quad \limsup _{n \rightarrow \infty}\left|\operatorname{Im} \alpha_{n}\right| \cdot\left|P_{n}\right|=0,
$$

Proof By Lemmas 3.2, 3.4, Proposition 3.1 and Main Proposition 3.1.

Theorem 3.1 Assumptions and notations as in Proposition 3.1. Then

(1) $\left(u_{n}, P_{n}\right)$ subconverge in $W^{1,2}$ modulo bubbles, i.e., in the limit, the necks contain no energy if and only if

$$
\liminf _{n \rightarrow \infty}\left|\operatorname{Re} \alpha_{n}\right| \cdot\left|P_{n}\right|=0
$$


(2) $\left(u_{n}, P_{n}\right)$ subconverge in $C^{0}$ modulo bubbles, i.e., in the limit, the images of the necks become points if and only if

$$
\liminf _{n \rightarrow \infty} \sqrt{\left|\operatorname{Re} \alpha_{n}\right|} \cdot\left|P_{n}\right|=0 .
$$

Proof (1) The result is a direct consequence of the identity (3.21) in Main Proposition 3.1. (2) “ $\Leftarrow$ ": If $\liminf _{n \rightarrow \infty} \sqrt{\left|\operatorname{Re} \alpha_{n}\right|} \cdot\left|P_{n}\right|=0$, then by Main Proposition 3.1 and passing to subsequences if necessary, we have $\lim _{n \rightarrow \infty} L\left(u_{n}, I_{n}^{i}\right)=0, i=0,1, \ldots, K$. On the other hand, by " $\epsilon$-regularity" and the fact that $\lim _{n \rightarrow \infty} \omega\left(u_{n}, I_{n}^{i}\right)=0, i=0,1, \ldots, K$, we get $\lim _{n \rightarrow \infty} \sup _{I_{n}^{i}}\left|\nabla u_{n}\right|=0, i=0,1, \ldots, K$. Here we used the fact that, after passing to subsequences, the local energy of $u_{n}$ over a neighborhood of the two boundary components of $I_{n}^{i}$ can be arbitrary small. Finally, applying Lemma 3.5, we conclude

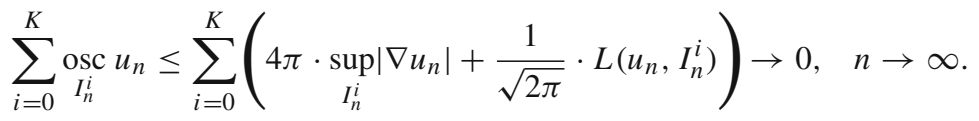

Thus, all necks converge to points in the target.

" $\Rightarrow$ ": If $\left(u_{n}, P_{n}\right)$ subconverges in $C^{0}$ modulo bubbles, then by the bubble and neck decomposition in Proposition 3.1 and passing to subsequences if necessary, we get

$$
\lim _{n \rightarrow \infty} \operatorname{osc}_{I_{n}^{i}} u_{n}=0, \quad i=0,1, \ldots, K .
$$

Hence, we have $u_{n}\left(I_{n}^{i}\right) \subset B\left(y_{i}, \rho_{i}\right)$ for some $y_{i} \in N$ with $\rho_{i}<\min \left(\frac{\pi}{2 \kappa}, \operatorname{inj}\left(y_{i}\right)\right)$, where $\kappa^{2}$ is an upper bound on the sectional curvature of $N$. Fix $i \in\{0,1, \ldots, K\}$ and write $I_{n}^{i}=\left[t_{n}^{1}, t_{n}^{2}\right] \times S^{1}$. Then the universal cover of $I_{n}^{i}$ is

$$
\widetilde{I}_{n}^{i}=\left\{(t, \theta) \in \mathbb{R}^{2}, t \in\left[t_{n}^{1}, t_{n}^{2}\right]\right\} .
$$

It is clear that $u_{n}: I_{n}^{i} \rightarrow B\left(y_{i}, \rho_{i}\right)$ lifts to a harmonic map

$$
\widetilde{u}_{n}: \widetilde{I}_{n}^{i} \rightarrow B\left(y_{i}, \rho_{i}\right) .
$$

Applying the interior gradient bound for harmonic maps [13,14], we get

$$
\left|d \widetilde{u}_{n}\left(x_{0}\right)\right| \leq c_{0} \max _{x \in B\left(x_{0}, R\right)} \frac{d\left(\widetilde{u}_{n}(x), \widetilde{u}_{n}\left(x_{0}\right)\right)}{R}
$$

provided $B\left(x_{0}, R\right) \subseteq \widetilde{I}_{n}^{i}$, where $c_{0}$ is a constant depending only on $N$. Let $t_{0}=\frac{t_{n}^{2}+t_{n}^{1}}{2}$ and take $x_{0} \in\left\{t_{0}\right\} \times \mathbb{R}$. Then $B\left(x_{0}, \frac{t_{n}^{2}-t_{n}^{1}}{2}\right) \subseteq \widetilde{I}_{n}^{i}$, and by (3.32), we have

$$
\left|d \widetilde{u}_{n}\left(x_{0}\right)\right| \leq \frac{2 c_{0}}{t_{n}^{2}-t_{n}^{1}} \cdot \underset{I_{n}^{i}}{\operatorname{osc}} \widetilde{u}_{n} .
$$

Hence, for $\left(t_{0}, \theta\right) \in\left\{t_{0}\right\} \times S^{1}$,

$$
\left|d u_{n}\left(t_{0}, \theta\right)\right| \leq \frac{2 c_{0}}{t_{n}^{2}-t_{n}^{1}} \cdot \underset{I_{n}^{i}}{\operatorname{osc}} u_{n} .
$$

It follows from Lemma 3.3 and Definition 3.1 that

$$
\sqrt{\left|\operatorname{Re} \alpha_{n}\right|}=\left.\left|\int_{\left\{t_{0}\right\} \times S^{1}}\right|\left(u_{n}\right)_{t}\right|^{2}-\left.\left|\left(u_{n}\right)_{\theta}\right|^{2} d \theta\right|^{\frac{1}{2}} \leq \frac{2 c_{0}}{t_{n}^{2}-t_{n}^{1}} \cdot \underset{I_{n}^{i}}{\operatorname{osc}} u_{n} .
$$


Multiplying by $\left|I_{n}^{i}\right|=\left|t_{n}^{2}-t_{n}^{1}\right|$ on both sides of (3.33) gives

$$
\sqrt{\left|\operatorname{Re} \alpha_{n}\right|} \cdot\left|I_{n}^{i}\right| \leq C_{0} \underset{I_{n}^{i}}{\operatorname{osc}} u_{n},
$$

where $C_{0}$ is a constant depending only on $N$, but not on $i$ and $n$. Summing up the inequalities on $I_{n}^{i}$ and applying (3.31), we get

$$
\sum_{i=0}^{K} \sqrt{\left|\operatorname{Re} \alpha_{n}\right|} \cdot\left|I_{n}^{i}\right| \leq C_{0} \sum_{i=0}^{K} \underset{I_{n}^{i}}{\operatorname{osc}} u_{n} \rightarrow 0, \quad n \rightarrow \infty .
$$

We thus conclude from Main Proposition 3.1 that

$$
\lim _{n \rightarrow \infty} \sum_{i=0}^{K} L\left(u_{n}, I_{n}^{i}\right)=\lim _{n \rightarrow \infty} \sqrt{\left|\operatorname{Re} \alpha_{n}\right|} \cdot\left|P_{n}\right|=\lim _{n \rightarrow \infty} \sum_{i=0}^{K} \sqrt{\left|\operatorname{Re} \alpha_{n}\right|} \cdot\left|I_{n}^{i}\right|=0 .
$$

Combining Proposition 3.1 and Main Proposition 3.1 gives the following:

Theorem 3.2 Assumptions and notations as in Main Proposition 3.1. Then there exist finitely many harmonic spheres $\omega^{j, l}: S^{2} \rightarrow N, j=1,2, \ldots, K ; l=1,2, \ldots, L_{j}$, such that after selection of a subsequence of $\left(u_{n}, P_{n}\right)$, we have

$$
\lim _{n \rightarrow \infty} E\left(u_{n}, P_{n}\right)=\sum_{j=1}^{K} \sum_{l=1}^{L_{j}} E\left(\omega^{j, l}\right)+\frac{1}{2} \lim _{n \rightarrow \infty}\left|\operatorname{Re} \alpha_{n}\right| \cdot\left|P_{n}\right| .
$$

\section{Harmonic maps from degenerating surfaces}

In order to study the compactness of a sequence of harmonic maps $u_{n}: \Sigma_{n} \rightarrow N$, we need to know how the domain surface varies. We collect some well-known facts about hyperbolic Riemann surface theory and refer to $[1,2,12]$ for more details.

Hyperbolic Riemann surfaces. We only consider surfaces without boundary. A Riemann surface $(\Sigma, c)$ is an orientable surface with a complex structure $c$. A hyperbolic surface $(\Sigma, h)$ is an oriented surface with a complete Riemannian metric $h$ of constant curvature -1 having finite area. The topological type of a surface is determined by its signature $(g, k)$, where $k$ is the number of punctures and $g$ is the genus of the surface obtained by adding a point at each puncture. The type $(g, k)$ is called general if

$$
2 g+k>2 \text {. }
$$

By the uniformization theorem, every Riemann surface of general type can be represented as a quotient $\mathbb{H} / \Gamma$, where $\mathbb{H}$ is the Poincaré upper half plane and $\Gamma$ is a Fuchsian group. Thus, it inherits a hyperbolic metric, where the punctures become ends. Conversely, for any hyperbolic surface $(\Sigma, h)$, the induced complex structure extends uniquely to a conformal structure on the compact surface obtained by adding a point at each puncture. In fact, there is a natural one-to-one correspondence between complex structures and hyperbolic metrics on surfaces of general type.

Two surfaces $\Sigma, \Sigma^{\prime}$ of type $(g, k)$ are called equivalent if there exists a conformal diffeomorphism $\Sigma \rightarrow \Sigma^{\prime}$ preserving the punctures (if there are any). The space of equivalence classes is called the moduli space $\mathcal{M}_{g, k}$ of Riemann surfaces of type $(g, k)$. The moduli 
space $\mathcal{M}_{g, k}$ in general has certain singularities and thus does not admit a $C^{\infty}$-structure. It has a covering space that is a manifold, namely the corresponding Teichmüller space. To this end, we fix a topological model surface $\Sigma_{0}$ of genus $g$ with $k$ punctures and then consider marked surfaces $(\Sigma, f)$, where $\Sigma$ is a Riemann surface of type $(g, k)$, and $f: \Sigma \rightarrow \Sigma_{0}$ is a homeomorphism preserving the punctures. Two marked surfaces $(\Sigma, f)$ and $\left(\Sigma^{\prime}, f^{\prime}\right)$ are called equivalent if there exists a conformal diffeomorphism $\Sigma \rightarrow \Sigma^{\prime}$ homotopic to $f^{\prime-1} \circ f$. The space of equivalence classes is called the Teichmüller space $\mathcal{T}_{g, k}$ of Riemann surfaces of type $(g, k)$.

Now we consider closed Riemann surfaces of genus $g>1$. Any such surface is of general type and it acquires a complete hyperbolic metric. Thus, we are working on the compactness of a sequence of harmonic maps whose domain surface $\Sigma$ varies in $\mathcal{M}_{g}$. Ideally, we hope the domain varies in a compact region. Unfortunately, the moduli space $\mathcal{M}_{g}$ is non-compact because the conformal structure on $\Sigma$ can degenerate. The following lemma [18] shows that the only process by which the conformal structure on $\Sigma$ can degenerate is the shrinking of simple closed geodesics on $\Sigma$. We represent $\Sigma$ as a quotient $\mathbb{H} / \Gamma$.

Lemma 4.1 Let $\left\{\Gamma_{n}\right\}$ be a sequence of Fuchsian groups which are isomorphic as abstract groups and with non-singular compact quotients $\mathbb{H} / \Gamma_{n}$. Suppose the lengths of simple closed geodesics on $\mathbb{H} / \Gamma_{n}$ are uniformly bounded from below by a positive constant. Then a subsequence of $\left\{\Gamma_{n}\right\}$ converges to some Fuchsian group $\Gamma$ which is isomorphic to all $\Gamma_{n}$. The convergence can be interpreted as the convergence of suitably normalized fundamental regions.

The natural way to compactify $\mathcal{M}_{g}$, then, is to allow the lengths of the geodesics to become zero and thus admit surfaces with nodes as singularities. Topologically, one cuts the surface at a collection of finitely many homotopically independent pairwise disjoint simple closed curves and pinches the cut curves to points. This yields the Deligne-Mumford compactification $\overline{\mathcal{M}}_{g}$, whose boundary $\overline{\mathcal{M}}_{g} \backslash \mathcal{M}_{g}$ consists of surfaces with nodes [5]. On $\mathcal{T}_{g}$, one can use Fenchel-Nielsen coordinates to describe this process and obtain the corresponding partial compactification $\overline{\mathcal{T}}_{g}$ (cf. [1,2]).

Here, following [12], we describe this process in terms of hyperbolic surface theory. Let $\Sigma_{0}$ be a topological model surface and $\mathscr{E}^{J}=\left\{\gamma^{j}, j \in J\right\}$ a possibly empty collection of finitely many pairwise disjoint, homotopically nontrivial, simple closed curves on $\Sigma_{0}$. Let $\widetilde{\Sigma}$ be the surface obtained from $\Sigma_{0}$ by pinching all curves $\gamma^{j}$ to points $\mathcal{E}^{j}$. We remove all $\mathcal{E}^{j}$ from $\widetilde{\Sigma}$ and place a complete hyperbolic metric $h$ on the resulting surface $\Sigma=\widetilde{\Sigma} \backslash \cup_{j \in J} \mathcal{E}^{j}$. For $j \in J$, we denote by $\left(\mathcal{E}^{j, 1}, \mathcal{E}^{j, 2}\right)$ a pair of punctures on $(\Sigma, h)$ corresponding to $\mathcal{E}^{j}$. Denote by $\bar{\Sigma}$ the surface obtained by adding a point at each puncture of $\Sigma$. Then the complex structure $c$ on $\Sigma$ that is compatible with the hyperbolic structure $h$ extends to a complex structure $\bar{c}$ on $\bar{\Sigma}$. $(\widetilde{\Sigma}, h, \bar{c})$ is called a nodal surface. $(\bar{\Sigma}, \bar{c})$ is called the normalization of $(\widetilde{\Sigma}, h, \bar{c})$ or $(\Sigma, h, c) . \bar{\Sigma}$ is a surface of lower topological type.

Let $\left(\Sigma_{n}, h_{n}, c_{n}\right)$ be a sequence of closed hyperbolic Riemann surfaces of genus $g>1$. We say that $\left(\Sigma_{n}, h_{n}, c_{n}\right)$ converges to a nodal surface $(\widetilde{\Sigma}, h, \bar{c})$ or a hyperbolic surface $(\Sigma, h, c)$, if there exist possibly empty collections $\mathscr{E}_{n}^{J}=\left\{\gamma_{n}^{j}, j \in J\right\}$ of finitely many pairwise disjoint simple closed geodesics on each $\left(\Sigma_{n}, h_{n}, c_{n}\right)$ and continuous maps $\tau_{n}: \Sigma_{n} \rightarrow \widetilde{\Sigma}$ with $\tau_{n}\left(\gamma_{n}^{j}\right)=\mathcal{E}^{j}$ for $j \in J$ and each $n$, such that:

(1) The lengths $\ell\left(\gamma_{n}^{j}\right)=l_{n}^{j} \rightarrow 0$ for all $j \in J$.

(2) $\tau_{n}: \Sigma_{n} \backslash \cup_{j \in J} \gamma_{n}^{j} \rightarrow \Sigma$ is a diffeomorphism for each $n$. 
(3) $\left(\tau_{n}\right)_{*} h_{n} \rightarrow h$ in $C_{l o c}^{\infty}$ on $\Sigma$.

(4) $\left(\tau_{n}\right)_{*} c_{n} \rightarrow c$ in $C_{l o c}^{\infty}$ on $\Sigma$.

By the thick-thin decomposition of a closed hyperbolic surface of genus $g>1$, the number of small simple closed geodesics (of lengths $<2 \operatorname{arcsinh}(1)$ ) is bounded by $3 g-3$ (cf. [12], Lemma IV.4.1). Thus, we have $0 \leq|J| \leq 3 g-3$. If $|J|>0$, we say $\left(\Sigma_{n}, h_{n}, c_{n}\right)$ degenerates to a nodal surface $(\widetilde{\Sigma}, h, \bar{c})$ or a hyperbolic surface $(\Sigma, h, c)$. Using our notations, we state the following proposition and refer to [12] for a detailed proof.

Proposition 4.1 Let $\left(\Sigma_{n}, h_{n}, c_{n}\right)$ be a sequence of closed hyperbolic Riemann surfaces of genus $g \geq 1$. Then, after selection of a subsequence, $\left(\Sigma_{n}, h_{n}, c_{n}\right)$ converges to a nodal surface $(\widetilde{\Sigma}, h, \bar{c})$ or a hyperbolic surface $(\Sigma, h, c)$.

Thus, the analysis of the degeneration of hyperbolic surfaces is reduced to the local behaviour of the pinched geodesics. A fundamental tool to realize this localization is the following collar lemma $[9,15,21,27]$. We again represent a closed Riemann surface of genus $g>1$ as a quotient $\mathbb{H} / \Gamma$.

Lemma 4.2 Let $\gamma$ be a simple closed geodesic of length $\ell(\gamma)=l$ in $\mathbb{H} / \Gamma$. Then there is a collar of area $\frac{l}{\sinh \left(\frac{l}{2}\right)}$ around $\gamma$, i.e., $\mathbb{H} / \Gamma$ contains an isometric copy of the region

$$
A=\left\{z=r e^{i \phi} \in \mathbb{H}: 1 \leq r \leq e^{l}, \arctan \left(\sinh \left(\frac{l}{2}\right)\right)<\phi<\pi-\arctan \left(\sinh \left(\frac{l}{2}\right)\right)\right\},
$$

where $\gamma$ corresponds to $\left\{r e^{i \frac{\pi}{2}} \in \mathbb{H}: 1 \leq r \leq e^{l}\right\}$, and the lines $\{r=1\},\left\{r=e^{l}\right\}$ are identified via $z \rightarrow e^{l} z$.

This collar neighborhood is a topological cylinder and its geometry is determined by the length of the core geodesic and is hence independent of the surface. There are other versions of the collar in terms of different coordinates, for example, a hyperbolic cylinder with Fermi coordinates [2]. In view of the results developed in Sect. 3, we need a standard cylindrical version of the collar (4.1). To this end, we consider the following conformal transformation:

$$
r e^{i \phi} \rightarrow(t, \theta)=\left(\frac{2 \pi}{l} \phi, \frac{2 \pi}{l} \log r\right) .
$$

Then the collar $A$ in Lemma 4.2 is isometric to the following cylinder:

$$
P=\left\{(t, \theta): \frac{2 \pi}{l} \arctan \left(\sinh \left(\frac{l}{2}\right)\right)<t<\frac{2 \pi}{l}\left(\pi-\arctan \left(\sinh \left(\frac{l}{2}\right)\right)\right), 0 \leq \theta \leq 2 \pi\right\}
$$

with metric

$$
d s^{2}=\left(\frac{l}{2 \pi \sin \frac{l t}{2 \pi}}\right)^{2}\left(d t^{2}+d \theta^{2}\right)
$$

here $\gamma \subset A$ corresponds to $\left\{t=\frac{\pi^{2}}{l}\right\} \subset P$, and the lines $\{\theta=0\},\{\theta=2 \pi\}$ in (4.3) are identified. 
Let $\operatorname{inj} \operatorname{rad}(\phi, r)$ be the injectivity radius at the point $(\phi, r)$ of $A$. Then, by results from hyperbolic trigonometry (see [12], Example 5.5 or [2], Chapter 2), one can verify that

$$
\sinh (\operatorname{injrad}(\phi, r)) \sin (\phi)=\sinh \left(\frac{l}{2}\right), \quad(\phi, r) \in A .
$$

Hence, applying the isometric transformation (4.2), we have

$$
\sinh (\operatorname{injrad}(t, \theta)) \sin \left(\frac{l t}{2 \pi}\right)=\sinh \left(\frac{l}{2}\right), \quad(t, \theta) \in P,
$$

where $\operatorname{inj} \operatorname{rad}(t, \theta)$ is the injectivity radius at the point $(t, \theta)$ of $P$.

Remark 4.1 (4.4) and (4.5) are very useful in that they give explicit expressions of the injectivity radius in terms of two different coordinates of the points in the collar.

Generalized energy identity. Consider a sequence of harmonic maps

$$
u_{n}:\left(\Sigma_{n}, h_{n}, c_{n}\right) \rightarrow N
$$

with uniformly bounded energy $E\left(u_{n}, \Sigma_{n}\right) \leq \Lambda<\infty$, where $\left(\Sigma_{n}, h_{n}, c_{n}\right)$ is a sequence of closed hyperbolic Riemann surfaces of genus $g>1$ with hyperbolic metrics $h_{n}$ and compatible complex structures $c_{n}$. We are only interested in the case that degeneration occurs. Thus, by Proposition 4.1, we can assume that $\left(\Sigma_{n}, h_{n}, c_{n}\right)$ converges to a hyperbolic Riemann surface $(\Sigma, h, c)$ by collapsing $p(1 \leq p \leq 3 g-3)$ pairwise disjoint simple closed geodesics $\gamma_{n}^{j}, j=1,2, \ldots, p$. Denote the $h_{n}$-length of $\gamma_{n}^{j}$ by $l_{n}^{j}$. Then in the degeneration $(n \rightarrow \infty)$, we have $l_{n}^{j} \rightarrow 0, j=1,2, \ldots, p$. For each $j$, the geodesics $\gamma_{n}^{j}$ degenerate into a pair of punctures $\left(\mathcal{E}^{j, 1}, \mathcal{E}^{j, 2}\right)$.

Proof of Theorem 1.1 We first consider the simpler case that $p=1$ and hence omit the indices $j$. Since $\lim _{n \rightarrow \infty} l_{n}=0$, w.l.o.g., we assume that $l_{n} \leq 2 \operatorname{arcsinh}(1)$ for all $n$. Let $P_{n}$ be the cylindrical collar about $\gamma_{n}$ given by (4.3) and let $\alpha_{n}=\alpha\left(u_{n}, P_{n}\right)$ be the complex number associated to $u_{n}$ along the collar $P_{n}$ as in Definition 3.1. After passing to a subsequence, we can assume that the limit

$$
\lim _{n \rightarrow \infty}\left|\operatorname{Re} \alpha_{n}\right| \cdot \frac{\pi^{2}}{l_{n}}
$$

exists in $[0, \infty]$. We will eventually see that the limit is finite, since the total energy of $u_{n}$ is uniformly bounded.

For $0<\delta<\operatorname{arcsinh}(1)$, let $\Sigma^{\delta}:=\{z \in \Sigma$, injrad $(z ; h) \geq \delta\}$ be the $\delta$-thick part of the hyperbolic surface $(\Sigma, h)$. Recall that there are diffeomorphisms $\tau_{n}: \Sigma_{n} \backslash \gamma_{n} \rightarrow \Sigma$ such that $\left(\left(\tau_{n}\right)_{*} h_{n},\left(\tau_{n}\right)_{*} c_{n}\right)$ converges to $(h, c)$ in $C_{l o c}^{\infty}$ on $\Sigma$. Set

$$
\bar{u}_{n}=\left(\tau_{n}\right)_{*} u_{n}, \quad \bar{h}_{n}=\left(\tau_{n}\right)_{*} h_{n}, \quad \bar{c}_{n}=\left(\tau_{n}\right)_{*} c_{n},
$$

and consider the following sequence of harmonic maps:

$$
\bar{u}_{n}:\left(\Sigma, \bar{h}_{n}, \bar{c}_{n}\right) \rightarrow N .
$$

Then for each fixed $\delta>0,\left(\bar{h}_{n}, \bar{c}_{n}\right)$ converges to $(h, c)$ in $C^{\infty}$ on $\Sigma^{\delta}$. Choose a fixed sequence $\delta_{n} \searrow 0$ such that $\Sigma^{\delta_{n}}$ exhaust $\Sigma$. Then by Theorem 2.2 and a standard diagonal argument, there exist finitely many blow-up points $\left\{x_{1}, x_{2}, \ldots, x_{I}\right\} \subset \Sigma$ which are away from the punctures $\left(\mathcal{E}^{1}, \mathcal{E}^{2}\right)$, finitely many harmonic maps $\sigma^{i, l}: S^{2} \rightarrow N, l=1,2, \ldots, L_{i}$, near the $i$-th blow-up point $x_{i}$ and a harmonic map $u:(\Sigma, h, c) \rightarrow N$ such that, after selection of a 
subsequence of $\left(u_{n}, \Sigma_{n}\right), \bar{u}_{n}:\left(\Sigma^{\delta_{n}}, \bar{h}_{n}, \bar{c}_{n}\right) \rightarrow N$ converges in $C_{l o c}^{\infty}$ to $u$ on $\Sigma$. $u$ extends smoothly to the normalization $(\bar{\Sigma}, \bar{c})$ of $(\Sigma, h, c)$. Moreover, the following holds:

$$
\lim _{n \rightarrow \infty} E\left(u_{n}, \tau_{n}^{-1}\left(\Sigma^{\delta_{n}}\right)\right)=\lim _{n \rightarrow \infty} E\left(\bar{u}_{n}, \Sigma^{\delta_{n}}\right)=E(u)+\sum_{i=1}^{I} \sum_{l=1}^{L_{i}} E\left(\sigma^{i, l}\right) .
$$

It should be remarked that the subsequence $\left(u_{n}, \Sigma_{n}\right)$ can be taken in such a way that $\lim _{n \rightarrow \infty} \operatorname{osc}_{\partial \Sigma^{\delta_{n}}} \bar{u}_{n}=0$, or equivalently, $\lim _{n \rightarrow \infty} \operatorname{osc}_{\partial\left(\Sigma_{n} \backslash \tau_{n}^{-1}\left(\Sigma^{\delta_{n}}\right)\right)} u_{n}=0$.

To recover the energy concentration at the punctures $\left(\mathcal{E}^{1}, \mathcal{E}^{2}\right)$, we have to study $\left(\bar{u}_{n}, \Sigma \backslash\right.$ $\left.\Sigma^{\delta_{n}}\right)$, or equivalently, $\left(u_{n}, \Sigma_{n} \backslash \tau_{n}^{-1}\left(\Sigma^{\delta_{n}}\right)\right)$. For each $n$ and $\delta, \Sigma_{n} \backslash \tau_{n}^{-1}\left(\Sigma^{\delta}\right)$ is not the $\delta$-thin part of $\left(\Sigma_{n}, h_{n}\right)$. However, we claim that for fixed small $\delta>0$ and for $n$ sufficiently large, $\Sigma_{n} \backslash \tau_{n}^{-1}\left(\Sigma^{\delta}\right)$ is almost the $\delta$-thin part of $\left(\Sigma_{n}, h_{n}\right)$.

To see this, fix $\delta>0$ small and let $z \in \Sigma$ be a point satisfying $\operatorname{injrad}(z ; h)=\delta$. Since $\left(\tau_{n}\right)_{*} h_{n}$ converges to $h$ in $C_{l o c}^{\infty}$ on $\Sigma$, then for all $\delta_{1}, \delta_{2}>0$ such that $\delta_{1}<\delta<\delta_{2}$, the following holds:

$$
\delta_{1}<\operatorname{injrad}\left(z ;\left(\tau_{n}\right)_{*} h_{n}\right)<\delta_{2}, \quad \text { for all } n \text { large enough. }
$$

Recall that for $0<\delta<\operatorname{arcsinh}(1)$, the $\delta$-thin part of a hyperbolic surface is either an annulus or a cusp (cf. [12], Proposition IV.4.2). For $n \geq 1$ and $\delta \in\left[\frac{l_{n}}{2}, \operatorname{arcsinh}(1)\right]$, let us see what the $\delta$-thin part of $\left(\Sigma_{n}, h_{n}\right)$ looks like. Recall that $P_{n}$ is the cylindrical collar about $\gamma_{n}$. Now, we define the following $\delta$-subcollars of $P_{n}$

$$
P_{n}^{\delta}:=\left[T_{n}^{1, \delta}, T_{n}^{2, \delta}\right] \times S^{1} \subseteq P_{n},
$$

where

$$
T_{n}^{1, \delta}=\frac{2 \pi}{l_{n}} \arcsin \left(\frac{\sinh \left(\frac{l_{n}}{2}\right)}{\sinh \delta}\right), \quad T_{n}^{2, \delta}=\frac{2 \pi^{2}}{l_{n}}-\frac{2 \pi}{l_{n}} \arcsin \left(\frac{\sinh \left(\frac{l_{n}}{2}\right)}{\sinh \delta}\right) .
$$

By (4.5), one can verify that $P_{n}^{\delta}$ is exactly the $\delta$-thin part of $\left(\Sigma_{n}, h_{n}\right)$, namely

$$
P_{n}^{\delta}=\left\{z \in \Sigma_{n}, \operatorname{injrad}\left(z ; h_{n}\right) \leq \delta\right\} .
$$

Thus, fix $\delta>0$ small, for all $\delta_{1}, \delta_{2}>0$ satisfying $\frac{l_{n}}{2}<\delta_{1}<\delta<\delta_{2}<\operatorname{arcsinh}(1)$, it follows from (4.8) and (4.11) that

$$
P_{n}^{\delta_{1}} \subseteq \Sigma_{n} \backslash \tau_{n}^{-1}\left(\Sigma^{\delta}\right) \subseteq P_{n}^{\delta_{2}}, \text { for all } n \text { large enough. }
$$

If we choose $\delta_{1}, \delta_{2}$ in (4.12) sufficiently close to $\delta$, then for $n$ large enough, $\Sigma_{n} \backslash \tau_{n}^{-1}\left(\Sigma^{\delta}\right)$ is almost the $\delta$-thin part $P_{n}^{\delta}$ of $\left(\Sigma_{n}, h_{n}\right)$. Thus we have verified our claim.

For $\delta>0$ small and for $n$ large enough, denote

$$
\Omega_{n}^{\delta}:=\left\{\left(\Sigma_{n} \backslash \tau_{n}^{-1}\left(\Sigma^{\delta}\right)\right) \backslash P_{n}^{\delta}\right\} \cup\left\{P_{n}^{\delta} \backslash\left(\Sigma_{n} \backslash \tau_{n}^{-1}\left(\Sigma^{\delta}\right)\right)\right\} .
$$

Note that $P_{n}$ are equipped with hyperbolic metrics which are conformal to $d t^{2}+d \theta^{2}$. By the conformal invariance of harmonic maps, we can replace the hyperbolic metrics with the metric $d t^{2}+d \theta^{2}$. Recall that $\lim _{n \rightarrow \infty} \operatorname{osc}_{\partial\left(\Sigma_{n} \backslash \tau_{n}^{-1}\left(\Sigma^{\delta_{n}}\right)\right)} u_{n}=0$. By applying " $\epsilon$-regularity" and taking subsequences, we have

$$
\lim _{n \rightarrow \infty} \operatorname{osc}_{\Omega_{n}^{\delta}} u_{n}=0, \quad \lim _{n \rightarrow \infty} E\left(u_{n}, \Omega_{n}^{\delta}\right)=0 .
$$

Thus, after passing to further subsequences, we conclude

$$
\lim _{n \rightarrow \infty} E\left(u_{n}, \Sigma_{n} \backslash \tau_{n}^{-1}\left(\Sigma^{\delta_{n}}\right)\right)=\lim _{n \rightarrow \infty} E\left(u_{n}, P_{n}^{\delta_{n}}\right) .
$$


Now the energy concentration at the punctures is reduced to the study of $\left(u_{n}, P_{n}^{\delta_{n}}\right)$. In view of (4.9) and (4.10), by choosing further subsequences of $\left(u_{n}, \Sigma_{n}\right)$, we have

$$
\left|P_{n}^{\delta_{n}}\right|=\frac{2 \pi^{2}}{l_{n}}-\frac{4 \pi}{l_{n}} \arcsin \left(\frac{\sinh \left(\frac{l_{n}}{2}\right)}{\sinh \delta_{n}}\right)=\frac{2 \pi^{2}}{l_{n}}(1+o(1)), \quad n \rightarrow \infty .
$$

To apply Theorem 3.2 with domain cylinders $P_{n}^{\delta_{n}}$, we see that the first two conditions, the "long cylinder property"(3.13) and the "uniform energy bound"(3.14), are satisfied. We need to check the "asymptotic boundary conditions"(3.15). For any fixed $R \geq 0$ and for fixed small $\delta>0$, denote

$$
A_{n}^{1}(\delta, R):=\left[T_{n}^{1, \delta}-1, T_{n}^{1, \delta}+R\right] \times S^{1}, \quad A_{n}^{2}(\delta, R):=\left[T_{n}^{2, \delta}-R, T_{n}^{2, \delta}+1\right] \times S^{1} .
$$

Then by (4.5), one can verify that the injectivity radii of the points in $A_{n}^{i}(\delta, R), i=1,2$, are uniformly bounded from below by a positive constant as $n \rightarrow \infty$. Hence the images $\tau_{n}\left(A_{n}^{i}(\delta, R)\right), i=1,2$ are uniformly away from the punctures of $\Sigma$. Moreover, the energies $\sum_{i} E\left(u_{n}, A_{n}^{i}(\delta, R)\right)$ can be uniformly controlled by $E\left(u, \Sigma \backslash \Sigma^{\delta^{\prime}}\right)$ (for some $\delta^{\prime}>\delta$ ), which goes to 0 as $\delta^{\prime} \rightarrow 0$. Thus, after passing to subsequences, one can verify the "asymptotic boundary conditions". Now, by Theorem 3.2, there exist finitely many harmonic maps $\omega^{k}$ : $S^{2} \rightarrow N, k=1,2, \ldots, K$, such that after selection of a subsequence, the following holds:

$$
\lim _{n \rightarrow \infty} E\left(u_{n}, P_{n}^{\delta_{n}}\right)=\sum_{k=1}^{K} E\left(\omega^{k}\right)+\lim _{n \rightarrow \infty}\left|\operatorname{Re} \alpha_{n}\right| \cdot \frac{\pi^{2}}{l_{n}} .
$$

Combining (4.7), (4.13) and (4.14) gives

$$
\lim _{n \rightarrow \infty} E\left(u_{n}\right)=E(u)+\sum_{i=1}^{I} \sum_{l=1}^{L_{i}} E\left(\sigma^{i, l}\right)+\sum_{k=1}^{K} E\left(\omega^{k}\right)+\lim _{n \rightarrow \infty}|\operatorname{Re} \alpha| \cdot \frac{\pi^{2}}{l_{n}} .
$$

Finally, we consider the general case $p>1$. By the thick-thin decomposition of hyperbolic surfaces (cf. [12], Lemma IV.4.1 and Proposition IV.4.2), both the short simple closed geodesics [of lengths $<2 \operatorname{arcsinh}(1)$ ] and the corresponding $\operatorname{arcsinh}(1)$-thin parts of the collars around them are pairwise disjoint. Hence we can deal with the corresponding subcollars separately, and the remaining proof is analogous to the simpler case. This completes the proof.

Proof of Theorem 1.2 W.l.o.g., we assume that $p=1$ and the $\operatorname{limit} \liminf _{n \rightarrow \infty} \sqrt{\left|\operatorname{Re} \alpha_{n}\right|} \cdot \frac{\pi^{2}}{l_{n}}$ exist in $[0, \infty]$. Then the results follow from applying Theorem 3.1 with domain cylinders $P_{n}^{\delta_{n}}$ as in the proof of Theorem 1.1.

Proof of Proposition 1.1 By Theorem 1.1 and Proposition 3.2.

Asymptotic behaviour. For each $j$, the asymptotic behaviour of the necks appearing near the $j$-th node is characterized by $\left\{\left(\alpha_{n}^{j}, l_{n}^{j}\right)\right\}_{n=1}^{\infty}$, namely

$$
E^{j} \approx\left|\operatorname{Re} \alpha_{n}^{j}\right| \cdot \frac{\pi^{2}}{l_{n}^{j}}, \quad L^{j} \approx \sqrt{\left|\operatorname{Re} \alpha_{n}^{j}\right|} \cdot \frac{\pi^{2}}{l_{n}^{j}},
$$

where $E^{j}$ is the sum of the energies of the necks and $L^{j}$ is the sum of the average lengths of the necks. In general, we have the following four cases as $n \rightarrow \infty$ : 
(1) $E^{j} \rightarrow E_{0}, \quad L^{j} \rightarrow \infty$,

(2) $E^{j} \rightarrow 0, \quad L^{j} \rightarrow \infty$,

(3) $E^{j} \rightarrow 0, \quad L^{j} \rightarrow L_{0}$,

(4) $E^{j} \rightarrow 0, \quad L^{j} \rightarrow 0$.

Here $E_{0} \in(0, \Lambda]$ and $L_{0} \in(0, \infty)$ are two constants.

Remark 4.2 (1) If $u_{n}:\left(\Sigma, h_{n}\right) \rightarrow(N, g)$ are conformal harmonic maps (i.e., minimal surfaces, in particular, pseudo-holomorphic curves [8]), i.e., $\Phi\left(u_{n}\right) \equiv 0$, then it is easy to verify that $\alpha_{n}^{j} \equiv 0$, for all $n$ and $j$. It follows immediately that

$$
\liminf _{n \rightarrow \infty}\left|\operatorname{Re} \alpha_{n}^{j}\right| \cdot \frac{\pi^{2}}{l_{n}^{j}}=0, \quad \liminf _{n \rightarrow \infty} \sqrt{\left|\operatorname{Re} \alpha_{n}^{j}\right|} \cdot \frac{\pi^{2}}{l_{n}^{j}}=0, \quad \forall j=1,2, \ldots, p .
$$

(2) If, in addition, we assume that $u_{n}$ is an energy-minimizing sequence in the same homotopy class, then, using a replacing argument from [4], one can show that in the limit the lengths of the necks are all finite, i.e., $\liminf _{n \rightarrow \infty} \sqrt{\left|\operatorname{Re} \alpha_{n}^{j}\right|} \cdot \frac{\pi^{2}}{l_{n}^{j}}<\infty$ for each $j$, which yields

$$
\liminf _{n \rightarrow \infty}\left|\operatorname{Re} \alpha_{n}^{j}\right| \cdot \frac{\pi^{2}}{l_{n}^{j}}=0, \quad \forall j=1,2, \ldots, p .
$$

(3) When the domain surfaces are degenerating tori, the problem is simpler for two reasons. Firstly, the moduli space of complex structures on the torus is simple. Secondly, any holomorphic quadratic differential on a torus is a constant. We refer to [40] for more details. It is worth mentioning that Parker's example [22] illustrates the asymptotics that the necks become longer and longer geodesics and carry a certain amount of energy. Some modifications to his example can illustrate the four cases of asymptotics mentioned, see [40].

Acknowledgments This paper is developed from a portion of the author's Ph.D. thesis [40]. He would like to thank his advisor, Prof. Jürgen Jost, for encouragement and inspiration. He would also like to thank Prof. Michael Struwe for conversations and advice, and Brian Clarke for discussions and help.

Open Access This article is distributed under the terms of the Creative Commons Attribution Noncommercial License which permits any noncommercial use, distribution, and reproduction in any medium, provided the original author(s) and source are credited.

\section{References}

1. Abikoff, W.: The real analytic theory of Teichmüller space. Lecture Notes in Mathematics, vol. 820. Springer, Berlin, (1980), vii+144 pp

2. Buser, P.: Geometry and spectra of compact Riemann surfaces. Progress in Mathematics, vol. 106, xiv+454 pp. Birkhäuser, Boston (1992)

3. Choi, H.I., Schoen, R.: The space of minimal embeddings of a surface into a three-dimensional manifold of positive Ricci curvature. Invent. Math. 81(3), 387-394 (1985)

4. Chen, J., Tian, G.: Compactification of moduli space of harmonic mappings. Comment. Math. Helv. 74, 201-237 (1999)

5. Deligne, P., Mumford, D.: The irreducibility of the space of curves of given genus. Inst. Hautes Études Sci. Publ. Math. 36, 75-109 (1969)

6. Ding, W.Y., Tian, G.: Energy identity for a class of approximate harmonic maps from surfaces. Comm. Anal. Geom. 3(3-4), 543-554 (1995)

7. Ding, W.Y., Li, J.Y., Liu, Q.Y.: Evolution of minimal torus in Riemannian manifolds. Invent. Math. 165(2), 225-242 (2006) 
8. Gromov, M.: Pseudo holomorphic curves in symplectic manifolds. Invent. Math. 82, 307-347 (1985)

9. Halpern, N.: A proof of the collar lemma. Bull. Lond. Math. Soc. 13, 141-144 (1981)

10. Hélein, F.: Régularité des applications faiblement harmoniques entre une surface et une varieté riemannienne. C. R. Acad. Sci. Paris Sér. I Math. 312, 591-596 (1991)

11. Hélein, F.: Harmonic Maps, Conservation Laws and Moving Frames. 2nd Edn. Cambridge University Press, Cambridge (2002)

12. Hummel, C.: Gromov's Compactness Theorem for Pseudo-Holomorphic Curves. Progress in Mathematics, vol. 151, viii+131 pp. Birkhäuser Verlag, Basel (1997)

13. Jost, J.: Two-Dimensional Geometric Variational Problems. Pure and Applied Mathematics (New York), x+236 pp Wiley-Interscience/Wiley, Chichester (1991)

14. Jost, J., Karcher, H.: Geometrische Methoden zur Gewinnung von a-priori-Schranken für harmonische Abbildungen. Manuscripta Math. 40(1), 27-77 (1982)

15. Keen, L.: Collars on Riemann surfaces. Discontinuous groups and Riemann surfaces. In: Proceedings of Conference, Universitty of Maryland, College Park, 1973. Annals of Mathematical Studies, vol. 79, pp. 263-268. Princeton University Press, Princeton (1974)

16. Lin, F., Wang, C.: Energy identity of harmonic map flows from surfaces at finite singular time. Calc. Var. Partial Differ. Equ. 6(4), 369-380 (1998)

17. Lamm, T.: Fourth order approximation of harmonic maps from surfaces. Calc. Var. Partial Differ. Equ. 27(2), 125-157 (2006)

18. Mumford, D.: A remark on Mahler's compactness theorem. Proc. Am. Math. Soc. 28, 289-294 (1971)

19. Minsky, Y.N.: Harmonic maps into hyperbolic 3-manifolds. Trans. Am. Math. Soc. 332(2), 607-632 (1992)

20. Minsky, Y.N.: Harmonic maps, length, and energy in Teichmüller space. J. Differ. Geom. 35(1), 151-217 (1992)

21. Matelski, J.A.: A compactness theorem for Fuchsian groups of the second kind. Duke Math. J. 43, 829840 (1976)

22. Parker, T.H.: Bubble tree convergence for harmonic maps. J. Differ. Geometry 44, 595-633 (1996)

23. Parker, T.H., Wolfson, J.: Pseudo-holomorpohic maps and bubble trees. J. Geometric Anal. 3(1), 63-98 (1993)

24. Qing, J.: On singularities of the heat flow for harmonic maps from surfaces into spheres. Comm. Anal. Geom. 3(2), 297-315 (1995)

25. Qing, J.: A remark on the finite time singularity of the heat flow for harmonic maps. Calc. Var. Partial Differ. Equ. 17(4), 393-403 (2003)

26. Qing, J., Tian, G.: Bubbling of the heat flows for harmonic maps from surfaces. Comm. Pure Appl. Math. 50(4), 295-310 (1997)

27. Randol, B.: Cylinders in Riemann surfaces. Comment. Math. Helv. 54, 1-5 (1979)

28. Rivière, T.: Conservation laws for conformally invariant variational problems. Invent. Math. 168, 1-22 (2007)

29. Sacks, J., Uhlenbeck, K.: The existence of minimal immersions of 2-spheres. Ann. Math. 113, 1-24 (1981)

30. Sacks, J., Uhlenbeck, K.: The minimal immersions of closed Riemann surfaces. Trans. Am. Math. Soc. 271, 639-652 (1982)

31. Struwe, M.: On the evolution of harmonic mappings. Comment. Math. Helv. 60, 558-581 (1985)

32. Topping, P.: Repulsion and quantization in almost-harmonic maps, and asymptotics of the harmonic map flow. Ann. Math. 159(2), 465-534 (2004)

33. Topping, P.: Winding behaviour of finite-time singularities of the harmonic map heat flow. Math. Z. 247(2), 279-302 (2004)

34. Wang, C.Y.: Bubble phenomena of Palais-Smale-like sequences from surfaces to general targets. Houston J. Math. 22(3), 559-590 (1996)

35. Wolf, M.: The Teichmüller theory of harmonic maps. J. Differ. Geom. 29(2), 449-479 (1989)

36. Wolf, M.: Infinite energy harmonic maps and degeneration of hyperbolic surfaces in moduli space. J. Differ. Geom. 33(2), 487-539 (1991)

37. Wolf, M.: High energy degeneration of harmonic maps between surfaces and rays in Teichmüller space. Topology 30(4), 517-540 (1991)

38. Wolfson, J.G.: Gromov's compactness of pseudo-holomorphic curves and symplectic geometry. J. Differ. Geom. 28(3), 383-405 (1988)

39. Ye, R.G.: Gromov's compactness theorem for pseudo-holomorphic curves. Trans. Am. Math. Soc. 342(2), 671-694 (1994)

40. Zhu, M.: Harmonic maps and Dirac-harmonic maps from degenerating surfaces. Ph.D. thesis, University of Leipzig (submitted) (2007) 